Itinéraires Itinéraires

Littérature, textes, cultures

\title{
Les performances d'improvisation de Tanya Tagaq : une analyse descriptive de la culture ethno-pop
}

\section{Sophie Stévance}

\section{(2) OpenEdition}

\section{Journals}

\section{Édition électronique}

URL : http://journals.openedition.org/itineraires/2765

DOI : $10.4000 /$ itineraires.2765

ISSN : 2427-920X

Éditeur

Pléiade

\section{Référence électronique}

Sophie Stévance, "Les performances d'improvisation de Tanya Tagaq : une analyse descriptive de la culture ethno-pop », Itinéraires [En ligne], 2015-1 | 2015, mis en ligne le 18 décembre 2015, consulté le 20 avril 2019. URL : http://journals.openedition.org/itineraires/2765; DOI : 10.4000/itineraires.2765

Ce document a été généré automatiquement le 20 avril 2019.

\section{(c) $(1)$}

Itinéraires est mis à disposition selon les termes de la licence Creative Commons Attribution - Pas d'Utilisation Commerciale - Pas de Modification 4.0 International. 


\title{
Les performances d'improvisation de Tanya Tagaq : une analyse descriptive de la culture ethno-pop
}

\author{
Sophie Stévance
}

\section{Contexte}

Les jeunes artistes autochtones d'aujourd'hui sont tout à la fois attachés à la préservation de leur patrimoine et portés par l'envergure transculturelle de leurs productions. Ils savent volontiers aller au-delà de leur patrimoine culturel inuit pour se nourrir d'influences diverses afin d'intégrer leurs créations à la production artistique transnationale (Meintel 1993). Tel qu'expliqué dans de précédents travaux (Chartier 2005), la chanteuse inuit Tanya Tagaq, comme d'autres artistes et écrivains amérindiens et inuits, affirme un «droit à la modernité » par le mélange culturel, le métissage entre des éléments locaux et empruntés. Tout comme l'artiste inuite Lucie Idlout (née en 1978), Tanya Tagaq, qui pratique une forme de chant de gorge, le katajjaq, dans un contexte de musique pop-électronique, défend son droit de «ne pas sonner indigène » (Diamond 2002), tout en faisant allusion à l'histoire de sa communauté. Cette revendication (qui apparait également dans les cultures scandinave, québécoise et inuite) serait somme toute favorisée par une tension qui s'exerce entre le général et le particulier, due au fait que la plupart de ces cultures, alors en marge de grands empires, se constituent dans la synthèse de leurs influences. Les modes d'intégration d'éléments de musique locale (ex. : le chant de gorge) et occidentale (ex. : les outils technologiques) dans la pratique musicale de Tagaq sont révélateurs de cette tension entre universel et particulier, global et local, collectif et singulier, et c'est au carrefour de ces jeux de polarité que Tagaq construit sa « signature singulière » (Desroches 2008). Comment ce dialogue culturel se manifeste-t-il dans ses prestations musicales? Quels aspects spécifiques de sa pratique artistique relèvent, d'une part, d'une pratique plus traditionnelle et, d'autre part, d'une pratique occidentale non autochtone? 
2 Pour mieux comprendre la pratique musicale improvisée de Tagaq, la présente contribution portera sur la manière dont la chanteuse construit ses improvisations sur la base du katajjaq traditionnel et de codes culturels et musicaux occidentaux. Tout d'abord, une réflexion sur la manière dont Tagaq utilise le katajjaq se pose en effet, car au cours des entretiens que j'ai eus avec elle, l'artiste me confiait considérer que sa pratique, bien qu'influencée par la tradition, n'a que très peu de choses à voir avec ce chant ancestral dans la mesure où elle improvise la plupart du temps. Que veut-elle dire ? La question se pose d'autant plus si l'on tient compte du fait qu'il y a effectivement de l'improvisation dans le katajjaq traditionnel. Qu'est-ce qui est alors improvisé, et qu'est-ce qui ne l'est pas dans les performances de la chanteuse? Qu'y met-elle d'elle-même, qu'invente-t-elle, véritablement, sur scène, et en direct? L'hypothèse est que, à l'instar du katajjaq traditionnel, les performances d'improvisation solistes de Tagaq font apparaître des liens de similarités entre des segments différents apparaissant à divers moments du processus de création. Pour saisir cette construction, on s'appuiera sur des transcriptions de quelques performances de la chanteuse ainsi que sur son discours recueilli lors d'entretiens. Précisons que la musique de Tagaq ne sera ici, au plan étic (c'est-à-dire d'un point de vue externe au contexte culturel), qu'un objet de transcription ${ }^{1}$ pour le chercheur afin de faire apparaître l'organisation implicite du matériau sonore et ainsi l'observer et le classifier en fonction de critères conceptuels et perceptuels. Certains éléments sont, en effet, "non notables", selon Serge Lacasse (2002), qui proposait d'ailleurs une notation de ceux-ci à l'aide de graphiques segmentaux et picturaux ${ }^{2}$. Ces modélisations seront ensuite analysées à partir d'une observation des motifs répétés et transformés. Ces traces seront comparées entre elles du point de vue de leur macrostructure et de leur microstructure respectives. Il sera alors possible de déduire de cette observation une première description de certains aspects du processus de création de Tagaq, ici défini par une relation génétique entre l'improvisé et le composé. Car à la suite de Gérard Genette (1994), une improvisation s'appuie toujours, soit sur un thème préexistant, soit sur un certain nombre de formules, lesquels excluent toute possibilité d'une invention absolue de chaque instant sans aucune structure d'enchaînement.

\section{Repères biographiques}

3 Tanya Tagaq est née dans la localité inuit de Cambridge Bay, ou Ikaluktutiak, dans le territoire du Nunavut au Canada, en 1975. À 15 ans, elle quitte le Nunavut pour s'établir avec sa famille à Yellowknife puis, dans les années 1990, elle décide d'aller étudier les arts visuels au Nova Scotia College of Art and Design; elle en sort diplômée en 1998 et se destine alors à une carrière d'artiste peintre et de professeur d'art. Installée à Halifax, elle découvre un vaste monde de musique nouvelle, incluant la musique électronique, la musique de danse et la musique techno, mais elle s'ennuie rapidement de sa culture maternelle, de ses racines, des sons de l'Arctique. Elle se met alors à étudier le chant guttural inuit à partir des cassettes audio que sa mère lui envoyait de sa province nordique du Canada. Elle commence à se produire sur de petites scènes locales, des festivals de la région, jusqu'à sa rencontre avec Björk, en 2001, qui l'invitera sur sa tournée Vespertine et à participer à son album Medúlla (2004). Sa carrière est ainsi propulsée. Elle lance son disque Sinaa (2005), presque entièrement a cappella sur lequel elle chante « Ancestors » en duo avec la star islandaise. Puis Auk/Blood (2008), album qui se distingue du précédent par une forte présence de l'électronique. 
4 En raison de ses origines, on associe souvent l'art de Tanya Tagaq à la seule pratique du jeu de gorge inuit. Comme l'explique très clairement Tagaq ${ }^{3}$, le katajjaq est, à l'origine, une exécution entre deux femmes placées face à face et très proches l'une de l'autre, se tenant par les bras ou les épaules (Montpetit et Veillet 1977 : 159-160, Charron 1978 : 246 ). À tour de rôle, chacune va émettre un son sur un rythme rapide et soutenu, et ce son sera imité, ou transformé par l'autre ${ }^{4}$. Il s'agit d'une véritable compétition, mais toujours dans l'esprit d'un jeu ${ }^{5}$ et « avec beaucoup de plaisir » (Beaudry 1978 : 38), dont le but est d'essouffler l'adversaire et de lui faire perdre son rythme. Pour Tagaq, les sons et les formules sonores utilisés, généralement graves et gutturaux, peuvent être l'évocation de paysages, d'odeurs de la terre, du sang, l'imitation des sons de la nature, des grognements d'animaux, de rires, de pleurs, ou même avoir une hauteur précise ; l'ensemble constitue alors une mélodie, rythmée avec ce léger mouvement de balancier du corps que l'on peut par exemple observer dans les performances en duo de Tagaq lorsqu'elle interprète un chant de gorge traditionnel. Cette alternance va être brusquement stoppée par le «fourire » (Ibid. : 41) de l'une des joueuses qui va alors se tromper ou ne pourra plus suivre la cadence (Montpetit et Veillet $1977: 160)^{6}$. Le jeu de gorge inuit est donc vécu comme une pratique ludique, mais les partenaires de jeu n'en sont pas moins estimées pour leur « endurance », leur « imagination » ou pour l'« efficacité » des sons produits (Beaudry 1978 : 39), notamment s'ils sont « inhabituels » (Ibid. : 41-42). Le but n'est donc pas tant de remporter la partie, mais, à travers un « esprit compétitif » (Ibid.: 39), de démontrer l'ampleur de son talent, de faire la «démonstration de compétence physique et esthétique [...] dont la bonne qualité est essentielle au plaisir de l'auditoire » (Ibid. : 40). En effet, à l'origine, certains sons pouvaient être considérés par l'assistance (alors exclusivement composée de femmes) (voir Cavanagh $1976: 46)$ comme plus « prestigieux» à exécuter que d'autres :

La fin du jeu de gorge est déterminée autant par le manque d'endurance d'une joueuse du point de vue physique (étouffement, essoufflement) ou psychologique (fou-rire) que par un manque de compétence (incapacité de suivre un tempo, d'imiter un rythme ou un timbre) ou de créativité (incapacité d'introduire des variantes connues ou d'en inventer de nouvelles). Inversement, le prestige impliqué par le fait d'être reconnue compétente à ce jeu dépend de sa capacité à surpasser les autres joueuses sur tous les plans, physiques ou esthétiques. (Beaudry 1978 : 41)

Comme on peut le constater dans ses entretiens et enregistrements sonores, Tanya Tagaq maîtrise la technique traditionnelle du jeu de gorge. Cependant, son art ne saurait se résumer à cette seule pratique : «Katajjaq remains my main influence, and the depth of my voice reflects it well, but what I do has nothing to do with tradition, most of the time I improvise » (Tagaq, cité par Bernard 2007). " My music isn't necessarily solely based on interpreting the land at home ${ }^{7}$. " Qu'est-ce que la chanteuse veut dire si l'on tient compte $\mathrm{du}$ fait que, dans tous les katajjait, une large place est faite à l'invention, à l'improvisation à partir de modèles (expiration et inspiration, sons voisés et non voisés, familles de rythmes, modèles intonatifs, et syllabes, comme l'expliquent les textes pionniers ${ }^{8}$, et même Tagaq ${ }^{9}$ ) :

vowels, consonants, or whole syllables are extracted as the basis for an abstract sound. [...] the following features must also be considered: (a) patterns (i.e., sequence and rhythm) of voiced and voiceless articulation and (b) patterns of inhalation and exhalation. Pitch distinctions are found in a few of the games. (Cavanagh $1976:$ 44-45)

6 Il est d'ailleurs intéressant de constater que Tagaq se fonde sur certains aspects de ce « répertoire » qu'elle a appris, ce qu'elle me confirmait lors d'un entretien : « I draw upon 
certain elements of traditional throat singing, and allow my creativity to do the rest. Also, traditional throat singing is done with two women, I sing alone ${ }^{10}$ ». Ainsi, lorsque Tagaq dit «laisser aller sa créativité ", parle-t-elle d'improvisation? Dans ses performances improvisées, quel matériau viendrait du katajjaq traditionnel et quel autre viendrait d'elle-même? Y a-t-il d'autres aspects du katajjaq que l'on pourrait déceler dans sa pratique? Comment cela s'organise-t-il ? Comment ces éléments se déploient-ils?

\section{Analyse structurelle}

7 Pour tenter de mieux comprendre l'improvisation selon Tagaq et son apport au katajjaq traditionnel, nous avons procédé à une comparaison entre les transcriptions que nous avons réalisées de plusieurs enregistrements audiovisuels où la chanteuse chante seule, sans le support de l'électronique - l'amplification du microphone mise à part. La première transcription est celle d'un enregistrement d'une performance au Théâtre de Modène lors du festival Altro Suono, le 10 mai 2011 ; la deuxième provient du congrès du 3 juillet 2011 $\mathrm{du}$ Women's Worlds. Une troisième performance sera convoquée, celle du festival d'Ostrava qui a eu lieu le 12 juillet 2008. Nous tenons également compte de deux de ses albums : Sinaa (2005) et Auk/Blood (2008) ${ }^{11}$.

Pour analyser nos transcriptions, nous nous sommes tout d'abord concentrée sur les variantes et les constantes de la pratique de Tagaq par rapport au jeu de gorge traditionnel. L'écoute et les transcriptions (descriptives) montrent plusieurs apports de l'artiste : elle le pratique seule, ce qui lui donne une plus grande latitude dans la durée de la performance globale et dans le développement des éléments. En effet, sur scène, les sessions d'improvisation peuvent dépasser les trente minutes, tandis que dans le contexte traditionnel, les deux partenaires peuvent difficilement durer plus de deux ou trois minutes - c'est un défi où chacune cherche à déstabiliser l'autre pour remporter la partie. Une autre variante concerne la combinaison de différents modes de production vocale: voix gutturale, premier et deuxième mode de phonation (mais toujours sur fond de chant de gorge). Chacune de ces techniques vocales imbriquées semble remplir une "fonction " musicale : une fonction mélodique (voix modale et de tête), et une fonction rythmique (voix gutturale). La «fonction » pourrait ici être comprise du point de vue de l'analyse fonctionnelle de Stéphane Roy (2003), laquelle s'intéresse aux interactions et aux relations entre les différentes articulations syntaxiques à l'intérieur d'une « œuvre ». En élargissant la terminologie de Roy à la lumière de notre objet d'étude, l'hypothèse serait que les performances d'improvisation solistes de Tagaq, tout comme le katajjaq traditionnel, présentent des liens de similarités entre des segments différents apparaissant à divers moments du processus de création. Ces liens sont notamment renforcés par des techniques vocales imbriquées qui semblent chacune remplir une fonction musicale. Toutefois, attribuer une "fonction» musicale à la mélodie et au rythme oblige à une certaine prudence, si l'on tient compte du fait qu'avec un point de vue occidental, on aura davantage tendance à décoder que le rythme est un accompagnement mis au service de la mélodie. C'est ce qu'explique le musicologue Philip Tagg: "The dualism between melody and accompaniment is one of the most common basic devices of musical structuration. [...] Accompaniment can only occur within a musical structure consisting of separate strands exhibiting different degrees of perceived importance» (Tagg $2000: 17)^{12}$. Il poursuit : «Eurovision Song Contest entries and rock 
numbers all use the melody/accompaniment dualism as a basic structuring device » (Ibid $.: 18)$.

9 En outre, les transcriptions (voir fig. 1 et fig. 2 en annexes) montrent que les improvisations de Tagaq à l'étude semblent épouser une forme musicale classique. Nous nous sommes concentrée sur les liens de similarités entre des segments apparaissant à deux ou plusieurs reprises, avec ou sans variation, lors du processus de production. Ces segments, qui peuvent constituer les cellules principales et génératives, sont déterminés en repérant, au sein d'une performance d'improvisation, le nombre de fois où la chanteuse répète une cellule (à l'identique ou partiellement), si elle revient à une séquence qui a déjà été énoncée ou si la chanteuse invente une nouvelle cellule. Comment repérer ces éléments? Cela peut, par exemple, porter sur la durée des séquences et des motifs, mais aussi sur l'alternance de sons voisés/non voisés, inspirés/ expirés (techniques utilisées pour le katajjaq telles que décrites par Cavanagh 1976 : 44-45 ${ }^{13}$ ), sur des rythmes particuliers, des contours intonatifs, des changements de registre, de timbre et de rythme, ou encore des morphèmes. Une fois ces unités perçues repérées, il s'agit d'observer leurs traits communs dans le traitement et le développement de leurs composantes, le renvoi à des unités similaires, et les contrastes qu'elles affichent entre elles.

10 Ainsi, la figure 1 (modélisation de la performance improvisée de Tagaq au Théâtre de Modène lors du Festival Altro Suono, le 10 mai 2011 : Performance $1^{14}$ ) fait entre autres ressortir des procédures de transformation (par chevauchement, permutation, ajout ou suppression), et des procédures de chevauchement d'un élément sur un autre, donnant ainsi lieu à une autre séquence. On retrouve pareilles procédures dans la performance 2 de la chanteuse donnée à l'occasion du congrès du 3 juillet 2011 du Women's Worlds (fig. 2) $)^{15}$.

11 Ces analyses présentent la cohérence structurelle du processus créateur de Tagaq, avec une construction de l'improvisation qui montre que la chanteuse crée en suivant les règles du katajjaq : en termes de techniques vocales, mais aussi d'improvisation puisqu'il y a de l'improvisation dans le katajjaq traditionnel, et même un sens de la construction qui pourrait s'apparenter à des formes occidentales (comme la chanson de forme rondo). Par exemple, pour la performance à Modène, on peut dégager la forme suivante (voir fig. 1) : Introduction - lettre A - lettre $B$ - citation d'une mélodie préexistante - Reprise de $B$ (transformé $\left.=\mathrm{B}^{\prime}\right)$ - Reprise de A (transformé $\left.=\mathrm{A}^{\prime}\right)$ - coda $\left(\mathrm{A} / \mathrm{B} / \mathrm{B}^{\prime} / \mathrm{A}^{\prime} /\right.$ coda $)$. Pour la performance au Women's Worlds, nous retrouvons une construction similaire: Introduction - lettre $\mathrm{A} / \mathrm{B} /$ citation (mélodie préexistante $=$ chant traditionnel) $/ \mathrm{A}^{\prime} / \mathrm{B}^{\prime} /$ A"/ coda.

12 En plus d'une macrostructure commune à ces différentes performances, on observe plus localement, sur le plan de la microstructure, un canevas, une sorte de modèle préexistant. En outre, dans cet extrait d'une performance réalisée lors du festival Colours of Ostrava, en $2008^{16}$, Tanya Tagaq expose un motif présent dans la Performance 2 (A", mesures 40-44) qu'elle semble placer dans différents états : 
Fig. 3. Transcription d'un extrait de la performance de Tanya Tagaq au festival Colours of Ostrava (2006)

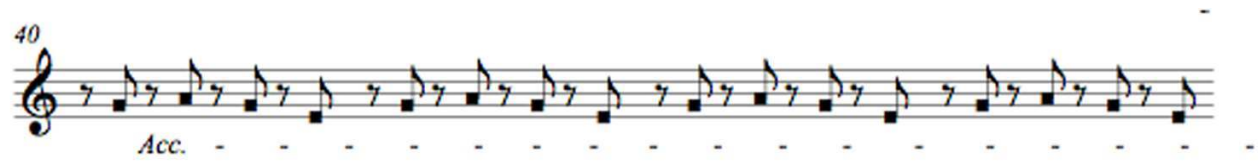
d'improvisation de Tanya Tagaq. Ce constat amène à envisager la possibilité que ses disques soient un matériau de base pour ses improvisations futures. En effet, nous possédons des «traces » du processus d'improvisation, lesquelles fournissent des indices en vue d'hypothèses. Tout d'abord, les modélisations affichent des similitudes de motifs que l'on retrouve tant sur les disques que lors des performances. Ceci montre l'importance du disque (en tant que matériau composé de départ qui pourra ensuite servir sur scène comme matière première à un discours plus improvisé) pour la performance d'improvisation de Tagaq. Ainsi, à la question : « How much of your music is improvised and how much is composed? Is it the same when you are recording versus playing live? ", Tagaq répondait : "90\% improvised, 10\% composed (Live). Recording is different, more like 50/50 because I have preset ideas before I begin recording ${ }^{20}$. " C'est aussi sans compter sur l'importance des objets audio grâce auxquels Tagaq a pu, plus jeune, apprendre les techniques du katajjaq traditionnel depuis les cassettes que sa mère lui envoyait. Il faut donc tenir compte de la force de l'écoute des cassettes audio sur l'apprentissage de la chanteuse de gorge en herbe.

15 L'objet audio a donc laissé des «traces » dans la pratique musicale de Tagaq, ces mêmes empreintes que l'on peut observer dans la musique pop ou chez d'autres improvisateurs, comme les musiciens de jazz, par exemple, qui cherchent, au cours de leur formation, à reproduire avec le plus d'exactitude possible les disques des jazzmen qu'ils prennent pour modèles. Ainsi, c'est en écoutant plusieurs enregistrements d'une même pièce que les musiciens de jazz apprennent cette pièce, et c'est en écoutant très attentivement les enregistrements d'autres musiciens que les improvisateurs se préparent à jouer avec d'autres musiciens. En jazz, les musiciens se constituent peu à peu une banque de motifs qu'ils répéteront et varieront au gré des divers moments d'improvisation. En revanche, en musique pop, le recours aux enregistrements est apparemment plus fidèle (voir Green 2007) - et cette utilisation des disques semble plus proche de celle de Tagaq.

\section{« Discomorphose »}

Ce phénomène est décrit par Antoine Hennion ${ }^{21}$ à travers l'idée de la " discomorphose ». Cette notion permet de penser les nouvelles modalités de l'écoute de la musique mises en place par le disque-de penser les comportements liés à la nature particulière de 
l'enregistrement. L'un de ces comportements est la recherche d'un degré de plus en plus élevé de haute-fidélité : plus on est exposé à des enregistrements de haute qualité sonore, plus on développe une compétence envers ces enregistrements sonores, ce qui mène à des attentes de la part des auditeurs. À la suite d'une écoute répétitive des disques, les auditeurs attendent, en effet, de l'interprète que son exécution en public soit aussi raffinée et précise que ce qu'ils ont entendu sur le disque. Cela a notamment pour conséquence une recherche effrénée de standards d'exécution. Ainsi, selon Hennion, avec le disque, «l'écoute se fait domestique, au double sens où elle s'effectue désormais majoritairement de façon solitaire, dans un espace privé, et où elle est apprivoisement d'un objet personnel, que l'on peut écouter à son rythme et qui vient meubler un intérieur » (1981: 146). Le sociologue montre également les traces de la discomorphose chez le mélomane, alors amené, par la force de l'écoute du disque, à comparer différentes interprétations d'un même morceau. Cet art phonographique a ainsi installé de nouvelles attitudes et habitudes d'écoute :

Ce format discographique est un phénomène tout à fait récent qui centre la musique sur la consommation, en général privée ou en tout petit groupe, chez soi ou dans ses espaces intimes, d'une musique dont on se sert «à volonté », dont on règle la dynamique, dont on recompose à son gré les séquences et la logique de répertoire. Toutes ces dimensions qui sont les traits banals de notre écoute actuelle constituent en réalité des réécritures fondamentales de ce qu'est notre relation à la musique. (Hennion 2000 : 19)

L'idée de la discomorphose présente l'influence de l'enregistrement, autant sur la pratique des musiciens (qui sont confrontés à des versions enregistrées de plus en plus précises) que sur les attentes des auditeurs (qui s'attendent à des niveaux de performance en concert au moins semblables à ce qu'ils entendent sur disque). Par conséquent, les sons et les sonorités entendus dans les versions enregistrées tendent à se constituer en tant que références: elles tendent à façonner l'oreille, et donc l'écoute du musicien autant que celle du mélomane. Cette distinction entre le disque et le concert est typique en musique pop, plus particulièrement dans les courants rock. C'est ce qu'a montré Serge Lacasse dans Groove. Enquête sur les phénomènes musicaux contemporains (2006). À travers les exemples de Deep Purple ou de Led Zeppelin, le musicologue observait une «divergence entre la version enregistrée et celle jouée sur scène » (Lacasse $2006: 75^{22}$ ) et l'impact de cet écart sur l'appréciation de la musique, ainsi que sur la discographie des groupes concernés, puisque l'album en concert devient partie intégrante du corpus musical pop. Il en est de même pour Tagaq : en octobre 2011 paraissait son album Anuraaqtuq, enregistré en concert lors $\mathrm{du} 26^{\mathrm{e}}$ Festival international de musique actuelle de Victoriaville (le 23 mai $2010^{23}$ ). Ceci montre également que les albums live - qui mettent en scène d'autres albums - font désormais partie intégrante de la discographie d'un artiste.

Suivant cette analyse, on peut déduire que la conception de Tagaq de la performance est proche de celle de la pop - c'est ici le signe d'une approche tout à fait «moderne » de la pratique du chant de gorge inuit, au-delà du fait de la pratique seule sur scène. Tagaq n'a toutefois pas entrepris de tournée en tant que telle pour présenter ses disques Sinaa ou Auk Blood, comme c'est, par exemple, le cas des groupes de musique pop, la plupart du temps dès la sortie d'un nouvel album afin d'en faire la promotion. À moins d'émettre l'hypothèse suivante : la performance de la chanteuse au Congress of Women's Worlds (Performance 2) pourrait-elle être une cover song en direct et sur scène de sa chanson «Surge ${ }^{24} »$ ? Plus systématiquement, pourrions-nous envisager ses performances d'improvisation comme des promotions non officielles de ses disques? Ainsi, et par 
exemple, sa performance présentée dans le cadre du festival Colours of Ostrava (Performance 3 : voir vidéo à 2'30) serait une version sur scène de sa chanson «Iglok » (figurant sur l'album collectif fRoots 20 : à 1'24) ${ }^{25}$. Il s'agit ici, suivant l'analyse de Serge Lacasse, d'une manière de diffuser ses œuvres sur scène, bien que Tagaq les présente comme des improvisations - et cela n'est pas contradictoire avec le discours de la chanteuse à propos de sa propre perception de ce qu'elle fait. Tagaq présenterait alors sur scène une cover song de ses compositions, avec une forte dose de spontanéité. À propos du trio qu'elle forme avec Jesse Zubot et Jean Martin, elle explique : « ce qui est intéressant [...] c'est l'imprévu auquel nous faisons face chaque soir. Nous ne savons jamais si le volume sera au maximum ou si la performance sera ténue au minimum. Nous sommes le reflet esthétique de ce qui nous habite ici et maintenant ${ }^{26}$. Où se passe alors l'imprévu dont parle Tagaq dans ses performances (en plus des paramètres qu'elle mentionne) ? Si la macrostructure qui ressort des analyses de nos modélisations reste sensiblement la même d'une performance à une autre (et d'un disque à l'autre), les transcriptions, l'analyse des enregistrements audiovisuels ainsi que l'analyse des disques ont tous montré une plus grande liberté en performance sur le plan de la durée de la performance globale et plus localement du développement des éléments sur scène - ce que ne permet pas le disque, dont la durée des chansons est limitée (en moyenne entre deux et trois minutes).

19 Ces analyses présentent la cohérence structurelle du processus de création de Tanya Tagaq, avec une construction de l'improvisation qui montre que la chanteuse crée non seulement en suivant les règles du katajjaq, mais en les dépassant pour répondre à certains standards formels de la scène pop-électro occidentale (voir Desroches, Stévance et Lacasse 2014).

\section{Conclusion}

L'objectif de notre contribution était de mieux comprendre l'un des aspects du processus de création de Tanya Tagaq. Nous avons ainsi proposé une méthode d'observation du processus de mise en ordre des éléments musicaux dans les performances solistes de la chanteuse, avec comme points focaux les constantes et les variantes dans les opérations d'écriture entre les différents paradigmes performanciels retenus. L'analyse des modélisations et de plusieurs enregistrements audiovisuels de performances d'improvisation de Tagaq en vue d'identifier des unités qui constituent chacune d'entre elles. Nous avons ainsi repéré, à l'intérieur de chaque performance analysée, les traits qu'elle partage avec d'autres. Ainsi, elle semble posséder une macrostructure semblable en raison d'une même conception des éléments constituant la microstructure - des cellules mélodiques répétées par transformation, des contours intonatifs ou des rythmes. On perçoit donc des constantes au sein des performances d'improvisation de Tanya Tagaq, qui s'échelonnent sur plusieurs années (2008 et 2011 pour les performances live; 2003-2008 pour les albums). Ces constantes ont été localisées et nous ont permis de dégager, chez la chanteuse, un modèle mélodico-rythmique implicite. Il s'agit donc d'un processus performanciel qui montre les étapes qui ont mené Tagaq à l'élaboration de son discours musical et l'assise de son langage musical ancré tout à la fois dans le katajjaq traditionnel (développement d'une cellule et répétition) et dans la culture occidentale avec une stratégie cognitive de la forme musicale globale (forme rondo ou forme ABA). 
21 À ce stade de l'analyse et à la suite de rencontres avec l'artiste, nous disposons d'indices précieux pour mieux comprendre la manière dont elle construit certaines de ses performances sur la base du katajjaq traditionnel et d'éléments occidentaux. Tagaq a intégré des schémas formels: "it's my bed », nous expliquait-elle en septembre 2013 lorsque nous lui avons présenté nos analyses. Nous pouvons alors tenter un premier constat, qui fera office de bilan. Ainsi, dans le processus créateur de Tanya Tagaq, il est possible de dégager une conception multiple de la performance d'improvisation musicale :

1. une improvisation typique du chant de gorge inuit traditionnel (avec tout ce que cela implique en termes de techniques vocales, d'images que les sons produits véhiculent, ainsi que l'idée de se dépasser avec mérite, une gradation des difficultés et un effort soutenu);

2. la performance d'improvisation s'appuyant sur une cohérence de la macrostructure en fonction d'une microstructure sémantique expérimentée - une base de texte chaque fois constituée d'une mélodie traditionnelle inuit préexistante et d'un certain nombre de formules qui se répètent par transformation. Dans ce cas, il s'agirait, « d'une phraséologie bien éprouvée, arpèges, gammes, bribes de citations au succès infaillible, qui contribuent fortement à ba(na)liser le parcours, pour l'auditoire comme pour l'interprète » (Genette $1982: 68)$;

3. la performance d'improvisation, considérée comme activité de lecture ouverte du disque, puisque Tagaq présente également sur scène une version de ses compositions enregistrées dans lesquelles elle introduit de légères variantes. Ainsi, la forme matérielle (le disque) fait la musique dans la mesure où le support d'écoute (le disque) permet l'activité. Pour l'instant, c'est donc de ses disques que découlent ses performances.

4. Mais on pourrait anticiper un autre état: la performance d'improvisation conçue comme une anticipation de ses compositions qu'elle déposera sur disque (autrement dit : ce qui sera mis sur disque proviendrait d'improvisations précédentes qu'elle a déjà réalisées sur scène).

Ces analyses ont permis de montrer la cohérence structurelle de ce processus créateur spontané dans ses caractéristiques les plus essentielles, intrinsèques et fécondes : avec un sens de l'improvisation qui montre, à la suite de Genette, que la chanteuse, « quel que soit son degré d'originalité et sa puissance d'invention, est toujours à la fois, dans l'instant, [créatrice] et interprète de sa propre création » (1982: 69). Sa pratique fait également ressortir un dialogue entre les aspects nordiques et occidentaux. Par exemple, les mélodies traditionnelles, donc les citations, les références, l'emploi de la technique du katajjaq, etc., et les aspects de la culture du Sud, occidentale (le disque, la construction par blocs, le microphone). C'est, à notre avis, dans la rencontre de ces deux cultures que s'étend l'espace de l'imaginaire musical de Tagaq. Du point de vue de la critique génétique, qui fait apparaître les aspects d'intratextualité dans l'activité performancielle de Tagaq à partir d'une lecture paradigmatique d'au moins deux de ses textes, sa performance d'improvisation s'apparente à un intertexte: puisque d'autres performances, d'autres œuvres, d'autres textes s'énoncent dans ses compositions/ improvisations « à des niveaux variables, sous des formes plus ou moins reconnaissables : les textes de la culture antérieure, ceux de la culture environnante », comme dirait Barthes (1973) ${ }^{27}$. Ainsi, «tout texte est un tissu nouveau de citations révolues », ce qui vaut pour la pratique performancielle de Tagaq ici analysée. C'est ainsi que l'intertextualité nous semble être un modèle opératoire et porteur pour saisir plus généralement le potentiel esthétique (c'est-à-dire l'ensemble des critères qui permettent à une même communauté de fonder un jugement partagé) qui se dégage du processus créateur de Tagaq. 
Il est possible d'approfondir la réflexion. Et de façon un peu analogue aux travaux de Serge Lacasse $(2005,2006 \mathrm{a}, 2006 \mathrm{~b}, 2010 \mathrm{a}, 2010 \mathrm{~b})$ qui portent sur la transphonographie en élargissant le modèle littéraire de la transtextualité proposé par Gérard Genette (1982) pour décrire les types de relations qui peuvent s'établir entre des enregistrements musicaux, nous proposons d'explorer la piste de l'intertextualité performancielle de Tanya Tagaq. Des analyses de nos transcriptions, plusieurs catégories peuvent être dégagées. Tout d'abord, l'intertextualité, qui a pour objet tout type de citation, ou d'allusion à d'autres textes allographiques, réfère à la pratique de Tagaq ici analysée lorsque la chanteuse introduit des éléments de «folklore », ce que j'ai appelé les mélodies préexistantes. Ensuite, si l'on s'intéresse à la façon dont Tagaq travaille pour transformer ses motifs, on se retrouve dans l'hypertextualité, correspondant à la transformation d'enregistrements déjà existants pour obtenir une autre œuvre par un processus de transformation. Or, lorsque Tagaq réutilise un motif, un fragment d'une œuvre qu'elle a composée (on parle ici d'autoréférence, d'autocitation), on plonge dans l'intratextualité. Le processus de création ici étudié témoigne encore de la polytextualité par la compilation de petits textes autonomes dans une plus grande structure. Enfin, la pratique de Tagaq nous parle d'architextualité, c'est-à-dire des relations génériques. Car chez Tagaq, les genres se chevauchent, à un point tel que ses œuvres peuvent appartenir à plusieurs genres musicaux à la fois, insinuant ainsi une incertitude quant au style auquel son œuvre devrait appartenir. Ces outils, qui sont des cas particuliers de l'intertextualité, semblent être un modèle opératoire et porteur car ils permettent de saisir plus généralement le potentiel esthétique et expressif qui se dégage du processus créateur de Tanya Tagaq.

Ainsi, Tagaq intègre dans son univers artistique autant d'éléments de sa culture inuite d'origine que de la culture transnationale; par transnationale, nous référons à des pratiques d'individus ne s'attachant pas à un territoire : ils les traversent au fil de leur vie en restant attachés, au plan émotif, symbolique et identitaire, à une terre d'origine (Meintel 1993; Chartier, Pepin et Ringuet 2006). À cet effet, nous avançons le terme d'ethno-pop pour décrire la singularité artistique de Tagaq. Somme toute préfigurée par des électrons libres comme le duo Kashtin, l'ethno-pop caractérise, selon nous, cette génération parvenue à inscrire sa musique dans la production culturelle mondiale. Car, et avec toute la réserve que la catégorisation impose, ce concept constitue une aide pour définir les contours d'un imaginaire collectif à travers l'observation de la production musicale et le discours d'une artiste, pour mieux comprendre comment cette production peut se situer dans le vaste champ de la création musicale d'aujourd'hui. Tandis que Karl Neuenfeldt (1996: 17) avance que la musique ethno-pop contemporaine des autochtones constitue un lieu important de critique culturelle, de protestation sociale et de résistance, nous envisageons plutôt l'ethno-pop comme un mouvement non restreint à la seule pratique musicale (par exemple: Samian, DJ Madeskimo, Shauit ou Taqralik Partridge) qui témoignerait de la manifestation d'un nouvel équilibre social et culturel. Nous pensons que ces artistes autochtones, dans une logique de réconciliation culturelle, ont su inscrire leur production artistique dans le mouvement actuel de globalisation, notamment par le biais de procédés de métissages culturels. Dans une perspective plus globale, nous proposons ainsi d'élargir le concept d'ethno-pop en l'abordant en tant que "scène ", au sens de William Straw (1991) ou, plus récemment, de Richard Peterson et Andy Bennett, pour qui le concept des scènes musicales « designate[s] the contexts in which clusters of producers, musicians and fans collectively share common tastes and 
collectively distinguish themselves from others" (2004: 1). De notre point de vue, l'ethno-pop, dont Tagaq est une digne représentante, montre que la jeunesse artistique autochtone fait, certes, émerger de nouvelles scènes et pratiques artistiques, mais participe surtout, d'égal à égal avec des artistes mondialement connus (par exemple Björk), à une production culturelle globale. En cela, l'ethno-pop se distingue de la « World music», concept habituellement utilisé par l'industrie du disque pour qualifier des pratiques musicales traditionnelles de diverses régions du monde rendues accessibles à un large auditoire occidental, et sous lequel les artistes comme Tagaq sont trop rapidement rangés. Or Tagaq et ces autres artistes cosmopolites font plutôt émerger un nouveau genre. L'ethno-pop nous permet, en effet, de penser et de caractériser la pratique artistique de Tagaq, paradigme de cette nouvelle vague d'artistes autochtones cosmopolites. Ce terme désigne des pratiques culturelles issues de membres de communautés isolées qui accèdent au réseau et à la reconnaissance artistique internationale dans le contexte d'un mouvement volontaire de conquête d'un nouvel équilibre entre les valeurs de leurs communautés d'origine et les valeurs transnationales.

\section{BIBLIOGRAPHIE}

Barthes, Roland, 1973, « Texte (théorie du) », Encyclopcedia universalis.

Beaudry, Nicole, 1978, « Le katajjaq un jeu inuit traditionnel », Études/Inuit/Studies, vol. 2, nº 1, p. 35-53.

Bernard, Yves, 2007, « Festival Pop Montréal - Le chant profond du Nord de Tanya Tagaq », Le Devoir, 4 octobre.

Berthiaume-Zavada, Claudette, 1980, « Pour l'adéquation de la transcription en ethnomusicologie : l'exemple du katajjaq », Canadian University Music Review / Revue de musique des universités canadiennes, $\mathrm{n}^{\circ} 1$, p. 35-46.

Brunet, Alain, 2011, «L'inconscient arctique de Tanya Tagaq », 6 février, http://

www.cyberpresse.ca/arts/musique/201102/06/01-4367549-linconscient-arctique-de-tanyatagaq.php, consulté en février 2014.

Cavanagh, Beverley, 1976, "Some throat games of Netsilik Eskimo women », Canadian Folk Music Journal, $\mathrm{n}^{\circ} 4$, p. 43-47.

-, 1981, « Review: Inuit Games and Songs. Chants et Jeux des Inuits », Ethnomusicology, vol. 25, $\mathrm{n}^{\circ}$ 2, p. 349-352.

Charron, Claude, 1978, « Toward Transcription and Analysis of Inuit Throat-Games : MicroStructure », Études/Inuit/Studies, vol. 2, n 1, 245-259.

Chartier, Daniel, 2005, « Définir des modernités hybrides. Entre société, patrimoine, savoir, pouvoirs contemporains et culture autochtones », Globe, Revue internationale d'études québécoises, vol. $8, \mathrm{n}^{\circ} 1$, p. $11-16$.

Chartier, Daniel, Pepin, Véronique et Ringuet, Chantal (dir.), 2006, Littérature, immigration et imaginaire au Québec et en Amérique du Nord, Paris, L'Harmattan. 
Crantz, David, 1767, The History of Greenland, Brethren's Society, [En ligne], https://archive.org/ stream/cihm_42578\#page/n241/mode/2up.

Crystal, David, 1975, The English Tone of Voice: Essays in Intonation, Prosody and Paralanguage, Londres, Arnold.

Delalande, François, 1976, « Pertinence et analyse perceptive », Cahiers Recherche/Musique, $\mathrm{n}^{\circ} 2$, p. 73-90.

Desroches, Monique, 1977, Ce qui s'est écrit sur la musique des Inuit : un bilan, mémoire de Maîtrise, Université de Montréal, Faculté de musique.

-, 2008, « Entre texte et performance : l'art de Raconter », Cahiers d'ethnomusicologie, $\mathrm{n}^{\circ} 21$, p. $103-115$.

Desroches, Monique, Stévance, Sophie et Lacasse, Serge, 2014, Quand la musique prend corps, Montréal, Presses de l'Université de Montréal.

Diamond, Beverley, 2002, « Native American Contemporary Music: The Women », Worlds of Music, vol. $44, n^{\circ} 1$, p. 9-35.

Eklund, Robert, « Pulmonic ingressive speech: a neglected universal? », [Présentation Powerpoint], http://www.ida.liu.se/ robek28/pdf/Eklund_2007_IngressiveSpeech_SLIDES.pdf.

Genette, Gérard, 1982, Palimpsestes. La littérature au second degré, Paris, Seuil.

-, 1994, L'Cuvre de l'art, Paris, Seuil.

Green, Lucy, [2001] 2007, How Popular Musicians Learn: A Way Ahead for Music Education, Farnham, Ashgate.

Gutzwiller, Andreas, 1979, « Stone Age and Promised Land: An Answer to James Reid », Ethnomusicology, vol. 23, $\mathrm{n}^{\circ}$ 1, p. 103-107.

Harvey, Denise, 1977, Analyse stylistique et comparative des jeux de gorge (Katajjait), mémoire de maîtrise, Université de Montréal.

Hennion, Antoine, 1981, Les Professionnels du disque, Paris, Métailié.

-, 2000, Figures de l'amateur. Formes, objets, pratiques de l'amour de la musique, Paris, La Documentation française.

Lacasse, Serge, 2005, « La musique comme discours phonographique : fondement d'une démarche d'analyse », Musicologies, $n^{\circ}$ 2, p. 23-39.

-, 2002, «Visual Representation of Sound Staging in Recorded Popular Music: Graphic Reference Tools », dans H. Terho et al. (dir.), Proceedings of the 2001 IASPM-International Conference, Turku, Finland, Turku, International Association for the Study of Popular Music, p. 778-787.

-, 2005, « La musique comme discours phonographique : fondement d'une démarche d'analyse », Musicologies, $\mathrm{n}^{\circ} 2$, p. 23-39.

,- 2006a, «Composition, performance, phonographie : un malentendu ontologique en analyse musicale? ", dans S. Lacasse et P. Roy (dir.), Groove. Enquête sur les phénomènes musicaux contemporains, Québec, Presses de l'Université Laval, p. 65-78.

,$- 2006 \mathrm{~b}$, «Stratégies narratives dans "Stan" d'Eminem : le rôle de la voix et de la technologie dans l'articulation du récit phonographique ", Protée, vol. 34, nº 2-3, p. 11-26.

-, 2010a, « Une introduction à la transphonographie », Volume!, vol. 7, nº 2, p. 31-55, [En ligne], http://volume.revues.org/692. 
-, 2010b, « The Phonographic Voice: Paralinguistic Features and Phonographic Staging in Popular Music Singing », dans A. Bayley (dir.), Recorded Music: performance, culture and technology, Cambridge, Cambridge University Press, p. 225-251.

Leard, Frank, 2008, L'Univers des musiciens : Analyse d'une expérience artistique, Toulouse, Presses universitaires du Mirail.

Meintel, Deirdre, 1993, « Transnationalité et transethnicité chez les jeunes issus de milieux immigrés à Montréal », Revue européenne des migrations internationales, vol. 9, nº 3, p. 63-79.

Montpetit, Carmen et Veillet, Céline, 1977, « Recherches en ethnomusicologie : les katajjait chez les Inuit du Nouveau-Québec », Études/Inuit/Studies, vol. 1, nº 1, p. 154-164.

Moore, Allan F., 1993, Rock: The Primary Text, Buckingham, Open University Press.

Nattiez, Jean-Jacques, 1983, « Some Aspects of Inuit Vocal Games », Ethnomusicology, vol. 27, n³ 3, p. 457-475.

-, 1999, «Inuit Throat-Games and Siberian Throat singing: a comparative, historical and semiological approach », Ethnomusicology, vol. 33, n 3, p. 399-418.

Neuenfeldt, Karl, 1996, « Songs of Survival. Ethno-Pop Music as Ethnographic Indigenous Media », Australian Canadian Studies, vol. 14, nº 1-2, p. 15-31.

Peterson, Richard et Bennett, Andy, 2004, Music Scenes: Local, Translocal, and Virtual, Nashville, Vanderbilt University Press.

Plastekes, George, 2005, « Re-flections on the Cover Age : A Collage of Continuous Coverage in Popular Music », Popular Music and Society, $\mathrm{n}^{\circ}$ 28, p. 137-161.

Prasad, Anil, 2010, « Tanya Tagaq, Instinctual Invocations », [En ligne], http:// www.innerviews.org/inner/tagaq.html.

Qureshi, Regula Burckhardt, 1987, « Music Sound and Contextual Input: A Performance Model for Musical Analysis », Ethnomusicology, vol. 31, nº 1, p. 56-86.

Rabau, Sophie, 2002, L'Intertextualité, Paris, Flammarion, GF-Corpus, Texte III.

Reid, James, «Transcription in a New Mode », Ethnomusicology, vol. 21, n 3, 1977, p. 415-433.

Robins, Robert H., 1967, A Short History of Linguistics, Londres, Longman Linguistics Library

Saladin d'Anglure, Bernard, 1978, «Entre cri et chant : les katajjait, un genre musical féminin », Études/Inuit/Studies, vol. 2, nº 1, p. 85-94.

Seeger, Charles, 1958, «Prescriptive and Descriptive Music Writing ", Musical Quaterly, vol. 44, $\mathrm{n}^{\circ}$ 2, p. 184-195.

Straw, William, 1991, "Systems of Articulation, Logics of Change: Scenes and Communities in Popular Music », Cultural Studies, vol. 5, n 3, p. 361-375.

Tagg, Philipp, 2000, « Melody and Accompaniment », Encyclopedia of Popular Music of the World, p. 1-19, [En ligne], http://www.andrelambert.org/uqam/principes/melodaccUS.pdf.

Tagaq, Tanya et Brunet, Alain, 2011, «L'inconscient arctique de Tanya Tagaq », 6 février, [En ligne], http://www.cyberpresse.ca/arts/musique/201102/06/01-4367549-linconscient-arctiquede-tanya-tagaq.php, consulté en février 2014. 


\section{Bibliographie complémentaire}

Crantz, Davis, 1767, The History of Greenland, Brethren's Society.

Beaudry, Nicole, 1978, « Toward Transcription and Analysis of Inuit Throat Games : Macrostructure ", Ethnomusicolgy, vol. 22, nº 2, p. 261-273.

Diamond, Beverley, 2011, « Medúlla, and: Sinaa (review) », Journal of American Folklore, vol. 124, $\mathrm{n}^{\circ}$ 491, p. 95-97.

Hennion, Antoine, 1993, La Passion musicale, Paris, Métailié.

Pelinski, Ramon, 1981, La Musique des Inuit du Caribou. Cinq perspectives méthodologiques, Montréal, Presses de l'Université de Montréal.

Stévance, Sophie, 2011, « The Inuit Katajjaq in Popular Culture: The Canadian Throat-Singer Superstar Tanya Tagaq », Itamar. Revista de Investigación Musical: Territorios para el Arte, $\mathrm{n}^{\circ} 3$, Valencia (Spain), p. 79-85.

- 2015, « La dimensione del "gioco" di gola inuit secondo Tanya Tagaq nella tradizione della nouvelle vocalité occidentale », dans Le dimensioni della voce, Besa Editrice, Nardò (Italy), p. 185-199.

Stévance, Sophie et Lacasse, Serge, 2015, « Research-creation in music as a Collaborative space between musicologist, researchers-creators, and musicians », Media- $N$, vol. 11, $\mathrm{n}^{\circ} 3$, [En ligne], http://median.newmediacaucus.org/research-creation-explorations/research-creation-inmusic-as-a-collaborative-space/.

\section{Discographie}

Björk, Medulla, 2004, Atlantic Recording Corporation (Warner). CD 62981.

Chants et jeux traditionnels inuit/ Inuit Traditional Songs and Games, 1984, Montreal, Radio-Canada SQN 108.

Idlout, Lucie, 2002, E5-770, My Mother's Name, Toronto ; Heart Wreck Records. AR 20432.

Muse, 2002, Hullabaloo Soundtrack, Taste Mushroom.

Muse, 2005, Absolution Tour, Warner.

Muse, 2007, HAARP, Warner Bross.

Tagaq, Tanya, 2003a, Tagaq/Deveau. Vocals : Tanya Tagaq Gillis, Turntables : DJ : Michel Deveau. Producer : Andy Maize. A \& R, MapleMusic Recordings. Pour écouter des extraits de l'album, voir http://www.ccca.ca/inuit/english/tanya.html?languagePref=en\&. Consulté sur Internet en septembre 2011.

,- 2003b, fRoots 20, Various artists, American Roots. Processed by : cddbd v1.5.2PL0. Copyright (c) Steve Scherf et al. : http://www.ccca.ca/inuit/english/tanya.html?languagePref=en\&; http:// cdtrrracks.com/bid506577.html

-, 2003c, Sonic Weave 2003. Pour une écoute en direct (prelisten track) : http://

www.getmp3muzik.com/Lyrics/2296774/Various-Artists/Sonic-Weave-2003-Radio-Promo/

Tanya-Tagaq-Creature/download-mp3/ [Désormais inaccessible].

-, 2005, Sinaa, Produced by Juan Hernandez. SS 13705 Valkeapää, Niko. 2004. Sierra. Oslo :

Duippidit, CD, DPP502. 
-, 2008, Auk/Blood, Produced by Juan Hernandez. Label JERICHO BEACH, JBM0801. Ipecac Recordings.

-, 2010, Anuraaqutug, with Jean Martin (percussion) and Jesse Zubot (violin, viola). Les Disques Victo. VICTO cd 121.

\section{Hyperliens}

http://grecem.oicrm.org.

http://grecem.oicrm.org/diffusions/.

http://grecem.oicrm.org/diffusions/tanya-tagaq-culture-ethno-pop/.

« Tanya Tagaq Gillis \& Celina Kalluk perform a traditional throat song », http://

grecem.oicrm.org/diffusions/tanya-tagaq-culture-ethno-pop/.

"Tanya Tagaq- Description of Throat Singing ", http://grecem.oicrm.org/diffusions/tanya-

tagaq-culture-ethno-pop/, à partir de la $6^{\mathrm{e}}$ minute.

\section{ANNEXES}

\section{Légende des transcriptions}

Nicole Beaudry, dans « Toward Transcription and Analysis of Iuit Throat Games: Macrostructure » (Ethnomusicolgy, vol. 22, 1978) a développé, à la suite des premières transcriptions de katajjait sur la portée traditionnelle par Denise Harvey (1977) et de celles effectuées par des chercheurs de l'Université Laval réunis sous la direction de Bernard Saladin D'anglure, une notation pour décrire le chant de gorge inuit. Partant de cette notation, j'ai ainsi tenté de représenter l'improvisation de Tanya Tagaq. Cependant, j'ai dû adapter la notation pour illustrer au mieux sa pratique étant donné les différentes utilisations vocales de Tagaq. J'ai ainsi opté pour des indications de timbre (voir Delalande 1976), de voix de tête (j'ai choisi l'abréviation commune «VT » pour alléger le texte et en faciliter la lecture), voix modale (VM), sons de gorges (SG), toutes placées sur une seule portée. À toutes fins utiles, je rappellerai, à la suite de Claudette BerthiaumeZavada (1980 : 47, note 5), que « les symboles relatifs aux sons voisés/non-voisés, expirés/ inspirés, ont été proposés par Nicole Beaudry (1978) et Claude Charron (1978), et utilisés extensivement pour la première fois par Denise Harvey dans son mémoire de maîtrise (1977) ».

Il ne s'agit donc pas d'une notation idéale pour qui considère que la partition doit être prescriptive (Seeger 1958). Il s'agit plutôt, dans mon cas, de partitions descriptives ; elles n'ont alors pas d'autre fonction que de « faire voir » en partie ce que Tagaq fait, en fonction de ce que le transcripteur perçoit à l'oreille - elle-même étant, d'ailleurs, très sélective, voire dé-formée par sa propre formation (voir Berthiaume-Zavada 1980 : 35-36). En outre, et tel que précisé dans mon texte, qu'est-ce qui me dit que la ligne que je considère comme étant la ligne mélodique n'a pas une autre fonction?

Transcription can never communicate music, but can communicate ideas about music. Even a bad recording gives a better impression of hichiriki music than the 
finest transcription can hope to convey to somebody who has never heard this instrument before. Transcription cannot successfully introduce an unknown musical style to the reader ; this must be done by performance. Transcription can, however, be a useful device in discussing certain aspects of music, and a prime criterion for its quality is whether it visually represents those aspects ${ }^{28}$. (Gutzwiller 1979 : 106)

Il ne faut donc pas prendre les présentes transcriptions au pied de la lettre (elles ne s'érigent pas en système), ceci afin d'éviter tout cloisonnement idéologique et d'autres habitudes esthétisantes traditionnelles où la partition est sacrée, intouchable. Cela dit, nos descriptions sont suffisamment claires pour permettre au lecteur d'accéder assez facilement à ce corpus musical et, dans leur ensemble, elles rencontrent les principaux critères établis par James Reid (suitability, accuracy, flexibility, utility, praticability, crosscultural applicability, universality) (Reid $1977: 416$ et 419-420).

Néanmoins, il est possible d'atteindre un autre niveau de caractérisation de la pratique de Tagaq par la réalisation de spectrogrammes, afin de montrer les traits distinctifs de sa pratique pour déterminer ce qui la singularise en tant que chanteuse de chant de gorge contemporain. On trouvera donc, en suivant cet hyperlien, un exemple de cette caractérisation.

\section{NOTES}

1. C'est ce que montre la grille d'analyse de Regula Qureshi Burckhardt (1987).

2. On mentionnera également Alan Moore (1993 : 31-32) qui propose un modèle de stratification par couches rythmique, mélodique, harmonique et strates de basse fréquence.

3. "Tanya Tagaq-Description of Throat Singing ", http://grecem.oicrm.org/diffusions/tanyatagaq-culture-ethno-pop/ à partir de la sixième minute.

4. Pour la description du katajjaq traditionnel, nous référons essentiellement aux études pionnières de Cavanagh (1976: 43-47), de Nicole Beaudry (1978), suivies de l'analyse critique approfondie de Monique Desroches (1977) concernant l'état de la recherche sur la littérature de la musique des Inuit sur ce sujet, de l'étude de Carmen Montpetit et Céline Veillet (1977) de l'équipe de Bernard Saladin d'Anglure à l'Université Laval (par exemple 1978), et de Claude Charron (1978). Par conséquent, on ne réfèrera pas aux textes qui se situent dans une perspective de compréhension différente de la pratique du katajjaq traditionnel, comme c'est notamment le cas de Nattiez (1983, 1999), lequel, en reprenant ou en reformulant les recherches de ses prédécesseurs, leur rendait alors, d'une certaine manière, un hommage explicite fort mérité. Dans le même ordre d'idées, concernant le disque de 1978 « Inuit games and songs. Chants et Jeux des Inuit. Canada» (Unesco Collection, Musical Sources), l'auteur de la recension - Beverley Cavanagh - rappelle subtilement et à juste titre que «It is especially fortuitous for the ethnomusicological community that the recordings, made in the field by Nicole Beaudry, Claude Charron, and Denise Harvey, are of such high audio quality and that, in the case of the katajjait, the stereo separation accurately represents the interchange of motives between the two performers of each game " (Cavanagh $1981: 350$ ).

5. Voir Nicole Beaudry (1978: 35-36) qui insiste sur cette dimension du jeu, à la suite de l'hypothèse émise par Beverley Cavanagh (1976:46).

6. Voir «Tanya Tagaq Gillis \& Celina Kalluk perform a traditional throat song ", http:// grecem.oicrm.org/diffusions/tanya-tagaq-culture-ethno-pop/.

7. Tanya Tagaq, citée par Prasad (2010), en ligne : http://www.innerviews.org/inner/tagaq.html. 
8. Charron $(1978$ : 253, 255). À la fin de son étude, Charron remercie d'ailleurs le linguiste Laurent Santerre (1935-1995), spécialiste de phonologie, lequel a joué un rôle essentiel dans l'étude des enregistrements sonores et dans la distinction entre son voisé et non voisé : " All the acoustical research and the spectrograms presented here were realized in collaboration with the Laboratoire de Phonétique Expérimentale of the Université de Montreal and its director, Professor Laurent Santerre. »

9. "Tanya Tagaq: démonstration de chants de gorge ", et "The sounds of throat singing": http://grecem.oicrm.org/diffusions/tanya-tagaq-culture-ethno-pop/.

10. Entretiens de Tanya Tagaq avec Sophie Stévance, mai 2011 et septembre 2013 à la Faculté de musique de l'Université Laval, Québec.

11. Pour visualiser ce corpus, nous renvoyons le lecteur au site web du Groupe de recherchecréation en musique : http://grecem.oicrm.org.

12. http://www.andrelambert.org/uqam/principes/melodaccUS.pdf.

13. Les différentes pratiques phoniques - par exemple exhalation, inhalation, son voisé ou non voisé - ont été conceptualisées et sont très répandues en linguistique, au moins depuis 1967 avec les travaux de Robert H. Robins (1967) ou ceux de David Crystal en paralinguistique (1975). Concernant les sons inhalés des « Esquimaux ", voir notamment les recherches de Robert Eklund (http://www.ida.liu.se/ robek28/pdf/Eklund_2007_IngressiveSpeech_SLIDES.pdf), lequel nous réfère d'ailleurs aux travaux du missionnaire David Crantz sur les «Eskimo female speech » où l'on apprend que le premier cas connu de voix inhalée remonte à 1765 (The History of Greenland, Brethren's Society, 1767 : https://archive.org/stream/cihm_42578\#page/n241/mode/2up).

14. http://grecem.oicrm.org/diffusions/tanya-tagaq-culture-ethno-pop/ ou http:// www.youtube.com/watch?v=nuCSck0YsUY. Ci-après mentionnée « Performance 1 ».

15. http://grecem.oicrm.org/diffusions/tanya-tagaq-culture-ethno-pop/ ou http:// www.youtube.com/watch?v=NxS6gbbJ9Vw. Ci-après mentionnée « Performance 2 ».

16. http://grecem.oicrm.org/diffusions/tanya-tagaq-culture-ethno-pop/ ou http:// www.youtube.com/watch? $=6991 \mathrm{zQm} 2$ Aeo. Voir notamment à 19' ou à 45'. Ci-après mentionnée «Performance 3 ».

17. Par exemple à 2'33.

18. Par exemple à 2'15.

19. Or l'utilisation de ce chant traditionnel par Tagaq a été décriée par des gens de son village : Tagaq a été accusée d'exploiter à des fins financières la tradition inuit (alors que l'artiste souhaitait, explique-t-elle, à travers son geste, diffuser l'art de sa culture). Comme le précise Beverley Diamond (2011: 95) : "Two "traditional Inuit songs" are acknowledged in the credits, one the popular throat song "Qimiruluapik," a growling representation of the sound of a puppy that has become something of a pièce de resistance for throat singers, and the other a drum dance song. "

20. Tanya Tagaq, citée par Geologist, 12 mars 2012 : http://www.brightestyoungthings.com/ music/geologist-interviews-tanya-tagaq/, [désormais indisponible], consulté en septembre 2012.

21. Frank Leard (2008: 35) écrit : "C'est ce qu'Antoine Hennion appelle la "discomorphose" de nos relations (poḯtiques et esthésiques) à la musique. »

22. J'ajoute que le groupe de rock britannique Muse s'évertue, à l'opposé, à produire sur scène une version la plus proche possible de l'album enregistré. La seule modification que nous relevons est un ralentissement du rythme par moments et une tonalité baissée au demi ton (nous renvoyons le lecteur aux enregistrements en public du groupe : Hullabaloo, en 2002, Absolution Tour, en 2005, et H.A.A.R.P., en 2008).

23. Tagaq, Tanya, 2010, Anuraaqutug, avec Jean Martin (percussion) et Jesse Zubot (violon, viola), Les Disques Victo. VICTO cd 121.

24. Une longue documentation existe au sujet des versions, mais nous renverrons principalement le lecteur aux travaux de Plastekes (2005). Le lecteur pourra également consulter directement 
l'article paru sur le site web suivant: http://www.popular-musicology-online.com/issues/02/ mosser.html.

25. Tanya Tagaq, 2003b, fRoots 20, Various artists, American Roots. Processed by: cddbd v1.5.2PL0. Copyright (c) Steve Scherf et al. (http://www.ccca.ca/inuit/english/tanya.html? languagePref=en\&) : http://cdtrrracks.com/bid506577.html (piste 18). Voir le site du GRECEM : http://grecem.oicrm.org/diffusions/.

26. Tagaq et Brunet (2011), http://www.lapresse.ca/arts/musique/201102/06/01-4367549linconscient-arctique-de-tanya-tagaq.php.

27. Voir Rabau (2002).

28. L'auteur cite également Fumio Koizumi: «Since the discrepancy between theory and performance has become wide, it is impossible to precisely designate a sequence of notes, or even the exact number of notes, to any mode. Actually, the prescribed notes found within any one composition (in a designated mode), will vary in pitch from instrument to instrument and/or voice » (note 4, p. 107).

\section{RÉSUMÉS}

La chanteuse inuit Tanya Tagaq, qui pratique une forme de chant de gorge (katajjaq) dans un contexte de musique pop-électronique, nous semble particulièrement représentative de toute une génération d'artistes autochtones. Comme Tagaq, des artistes comme Lucie Idlout, Elisapie Isaac ou Celina Kalluk, dépassent volontiers leur aire culturelle pour se nourrir d'influences diverses afin d'intégrer leurs créations à la production artistique transnationale. Quels aspects spécifiques de la pratique artistique de Tagaq relèvent, d'une part, d'une pratique plus traditionnelle et, d'autre part, d'une pratique contemporaine? Comment ce dialogue se manifeste-t-il dans les prestations scéniques de la chanteuse? Pour mieux comprendre le comportement esthétique de Tagaq, nous tiendrons tout d'abord compte de la manière dont elle construit ses improvisations sur la base du katajjaq traditionnel et de codes culturels et musicaux occidentaux. Une fois observés, ces éléments nous parlent de la construction identitaire de la jeune génération autochtone, dont la création multidisciplinaire et transnationale nourrit et reflète ce mouvement volontaire, non pas d'une quête, mais d'une conquête d'un nouvel équilibre culturel. L'ethno-pop nous permet de rendre compte de ce dialogue constant entre deux pôles d'attraction caractéristiques du profil cosmopolite de Tagaq, soit ses origines inuites et son intégration sur la scène contemporaine transnationale.

Inuk singer Tanya Tagaq, who practices a form of throat singing called "katajjaq" in a popelectro-experimental musical context, is likely to be representative of a whole generation of Aboriginal artists. Just like Tagaq, artists such as Lucie Idlout, Elisapie Isaac or Celina Kalluk go beyond their cultural range to feed themselves with various influences in order to integrate their creations to the transnational artistic production. Which specific aspects of Tagaq's practice reveal, on the one hand, a more traditional practice and, on the other, a contemporary practice? How does this cultural dialogue manifest itself on stage? To better understand her aesthetic behaviour, we will consider how Tagaq builds her improvisations, based on traditional katajjaq and on Occidental musical and cultural codes. Once observed, these elements express the identity building of the young Aboriginal generation, whose multidisciplinary and transnational creation enriches and reflects this voluntary movement, not as a quest but as a conquest for a new 
cultural balance. This constant dialogue between her inuk roots and her integration on the transnational contemporary scene (two poles of attraction defining Tagaq's cosmopolitan profile), is well served by ethno-pop.

INDEX

Mots-clés : Tanya Tagaq, ethno-pop, cosmopolitisme esthétique, katajjaq, performances d'improvisation, analyse performancielle

Keywords : Tanya Tagaq, ethno-pop, aesthetic cosmopolitanism, katajjaq, improvisatory performances, performance analysis

\section{AUTEUR}

\section{SOPHIE STÉVANCE}

Chaire de Recherche du Canada en recherche-création en musique, Professeur agrégée de musicologie, Faculté de musique, Université Laval, Directrice du Laboratoire de recherche-création en musique et multimédia (LARCEM) et du Groupe de recherche-création en musique (www.grecem.oicrm.org) 